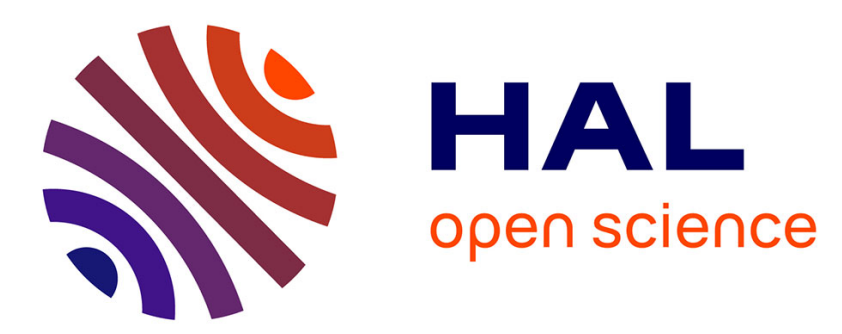

\title{
Role of Electron-Induced Coulomb Interactions to the Total SEU Rate During Earth and JUICE Missions
}

Pablo Caron, Christophe Inguimbert, Laurent Artola, Françoise Bezerra, Robert Ecoffet

\section{- To cite this version:}

Pablo Caron, Christophe Inguimbert, Laurent Artola, Françoise Bezerra, Robert Ecoffet. Role of Electron-Induced Coulomb Interactions to the Total SEU Rate During Earth and JUICE Missions. IEEE Transactions on Nuclear Science, 2021, 68 (8), pp.1607-1612. 10.1109/TNS.2021.3082573 . hal-03329133

\section{HAL Id: hal-03329133 \\ https://hal.science/hal-03329133}

Submitted on 30 Aug 2021

HAL is a multi-disciplinary open access archive for the deposit and dissemination of scientific research documents, whether they are published or not. The documents may come from teaching and research institutions in France or abroad, or from public or private research centers.
L'archive ouverte pluridisciplinaire HAL, est destinée au dépôt et à la diffusion de documents scientifiques de niveau recherche, publiés ou non, émanant des établissements d'enseignement et de recherche français ou étrangers, des laboratoires publics ou privés. 


\title{
Role of Electron-induced Coulomb Interactions to the Total SEU Rate during Earth and JUICE Missions
}

\author{
P. Caron, C. Inguimbert, L. Artola, F. Bezerra, R. Ecoffet
}

\begin{abstract}
The contribution of Coulomb processes induced by incident electrons in SEU sensitivity is investigated. The case of a 65-nm SOI memory is studied. Several orbits (GPS, JUICE's orbits) are analyzed. The relative importance to the total SEU rate of electron, proton and gamma environments is compared. SEU induced by electrons are obtained during Earth missions. In the environment of Jupiter, equivalent SEU sensitivities have been obtained between the studied device and the ESA SEU monitor. However, the relative importance of the physical processes involved is very different. Proton-induced SEU rates are estimated about one decade greater than electron-induced SEU rates. The Bremsstrahlung contribution has found negligible in such environment.
\end{abstract}

Index Terms - Space Environment, Single Event Upset, SOI memory, electron, proton.

\section{INTRODUCTION}

$\mathrm{E}$ lectron-induced SEU (Single Event Upset) sensitivity was first studied by M. P. King et al. [1]. Then, many studies demonstrated the ability of high-energy electrons to induce upsets in integrated memories [2]-[6]. More recently, some studies have detailed the different physical mechanisms involved in an electron environment [7]-[9]. It has been shown that for devices associated with low critical charges, the Coulomb processes (ionization and elastic scattering) are the main processes for triggering upsets. On the other hand, for devices associated with larger critical charges, it has been demonstrated that elastic Coulomb processes are dominated by electronuclear reactions [10], [11].

Electrons, in addition to Coulomb and electronuclear interactions, can generate photons through the Bremsstrahlung process. Thus, electron environments can produce secondary

Manuscript received October 12, 2020; revised February 07, 2021; accepted April 30, 2021.

P. Caron is with ONERA-DPHY, 2 avenue E. Belin, 31055 Toulouse, France (email: Pablo.Caron@onera.fr)

C. Inguimbert is with ONERA- DPHY, 2 avenue E. Belin, 31055 Toulouse, France (email: Christophe.Inguimbert@onera.fr) .

L. Artola is with ONERA- DPHY, 2 avenue E. Belin, 31055 Toulouse, France (email: Laurent.Artola@ onera.fr)

F. Bezerra is with CNES, 18 avenue E. Belin, 31401 Toulouse cedex 9, France (email: Francoise.Bezerra@cnes.fr)

R. Ecoffet is with CNES, 18 avenue E. Belin, 31401 Toulouse cedex 9, France (email: Robert.Ecoffet@ cnes.fr) photon environments. The flux of the photons generated depends, of course, on the flux of the primary electrons but also on the thickness of the shielding surrounding the device.

The greater the shielding thickness, the more photons will be generated. The study of M. Tali et al. [11], motivated by the JUICE mission, has shown that in the case of the ESA SEU monitor and for a large shielding thickness, electroninduced Bremsstrahlung is the main contributor for triggering upsets (considering the electron and proton environments encountered during the JUICE mission). The photons generated will in turn produce enough charges mainly thanks to photonuclear reactions. The ESA SEU monitor is a relatively large technology $(250 \mathrm{~nm})$ and has a large critical charge of about $10 \mathrm{fC}$. Our study analyzes the case of a more integrated device associated with a lower critical charge : the 65-nm SOI (Silicon On Insulator) SRAM (Static Random Access Memory) of IBM [12]. With this kind of device (low critical charge), electronuclear reactions are expected to be negligible compared to Coulomb interactions (ionization and elastic scattering). The consequences on the SEU rate calculations will be investigated. Proton-induced SEU sensitivity and electron-induced SEU sensitivity will also be compared.

To carry out our study, the sensitivity of the IBM device will have to be simulated in electron, proton and gamma environments. GPS and JUICE's orbits will be used to compare the relative severity of these environments. To conduct our analysis, GEANT4, a Monte-Carlo radiation transport tool [13], [14] is used (version 10.3 patch 03).

As a first step, the sensitivity of the IBM device in a proton environment will be studied. Experimental measurements are available [12] and will allow to validate our dedicated GEANT4 application. Then, the sensitivity of the device will be simulated for electron and gamma environments. Finally, the importance (in terms of SEU sensitivity) of electroninduced Coulomb interactions will be discussed for different orbit missions.

\section{SEU SENSITIVITY}

\section{A. RPP method}

As the relevance of the RPP (Rectangular Parallelepiped) approach has already been shown in [2], [7]-[9], [15] this approach has been chosen to evaluate the on-orbit SEU rate in this paper. In this approximation, the sensitive regions are 
assumed to be parallelepipeds with a given sensitive surface and a given sensitive thickness. An upset is caused if the deposited charge in the parallelepipeds is greater than the critical charge of the device.

As the 65-nm SOI SRAM of IBM [12] has been extensively studied by several authors [9], [12], [16], it has been selected for our study. All of its RPP parameters have already been defined, as summarized in Table I.

A relatively low critical charge can be noticed $(0.3 \mathrm{fC})$ which is equivalent to a critical energy of $6.75 \mathrm{keV}$.

TABLE I

RPP PARAMETERS OF THE IBM DEVICE

\begin{tabular}{ccc}
\hline Surface & Thickness & Critical charge \\
$0.1225 \mu \mathrm{m}^{2}$ & $60 \mathrm{~nm}$ & $0.3 \mathrm{fC}$ \\
\hline
\end{tabular}

Surrounding materials need to be accounted for in addition to the sensitive volumes. The structure used during the simulations was a simplified representation. The overlayers were assumed to be silicon layers. The dimensions of the structure were determined as explained in [15] and a thickness of about 5 microns was applied.

In addition, to perform accurate simulations of the deposited energy, the MicroElec module of GEANT4 was used [17], [18]. Based on the dielectric theory, the transport of electrons and protons is performed down to very low energy.

\section{B. Proton-induced SEU}

A full set of experimental data are available for the investigated device [12] allowing the comparisons with our GEANT4 simulations. Fig. 1 presents a comparison between the results obtained from GEANT4 simulations and the experimental data.

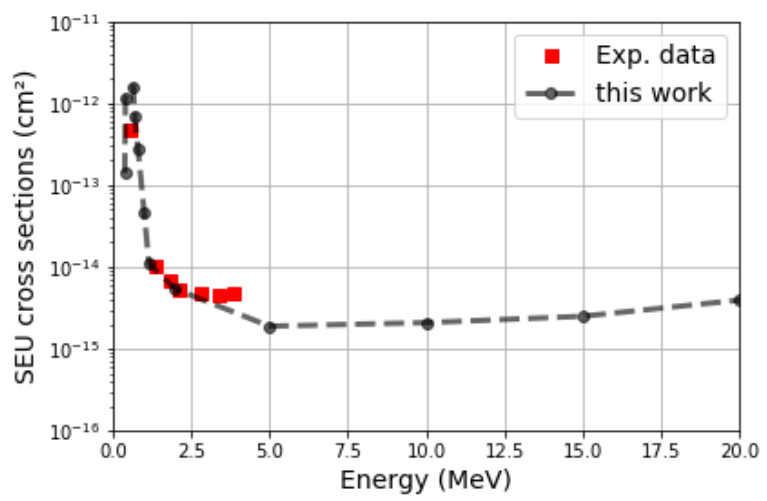

Fig. 1. Comparison of experimental measurements of proton-induced SEU [12] and GEANT4 simulations.

The ionization peak can clearly be observed for the lowest energies. An intermediate region is also identifiable after the ionization contribution and up to $3-4 \mathrm{MeV}$. Similarly to what has been already shown for other devices [9], [10], [15], this SEU region is mainly dominated by the elastic Coulomb process. Beyond $20 \mathrm{MeV}$, proton-induced SEU cross sections are roughly constant (near to $6 \cdot 10^{-15} \mathrm{~cm}^{2} / \mathrm{bit}$ ). As in [9], by using the RPP parameters presented in Table I, very good agreements can be found between our simulations and the experimental results.

\section{Electron-induced SEU}

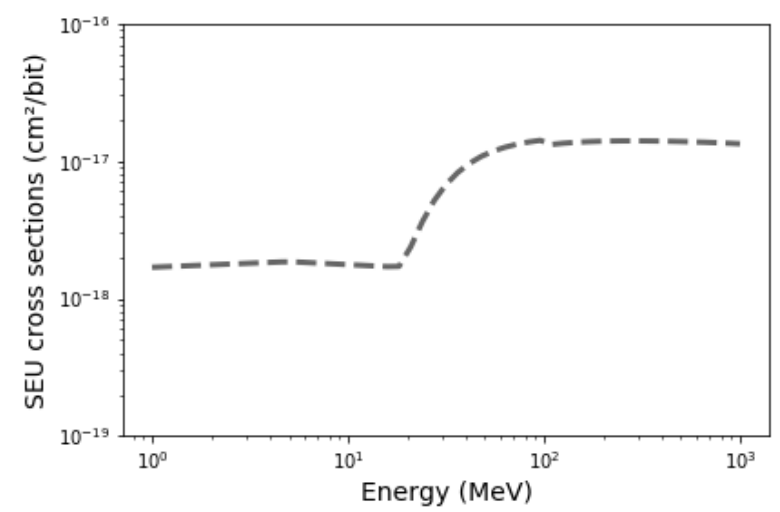

Fig. 2. Predictions of electron-induced SEU cross sections as a function of incident electron energies.

Electron sensitivity has been extrapolated by using the same GEANT4 application as used during proton simulations (see Fig. 1). Thus, the same RPP parameters are used. Predictions of electron-induced SEU cross sections are presented in Fig. 2. As explained in [7], [8] for other devices, the lower energy part of the curve (Fig. 2) is dominated by the direct ionization process. Above about $20 \mathrm{MeV}$, the contribution of elastic Coulomb reactions becomes the main contributor to the SEU cross sections [9], [15]. For such devices (associated with low critical charges), electronuclear contributions are negligible.

The ability of electrons to trigger upsets is several decades lower than that of protons (Fig. 1 vs. Fig. 2). Thus, for radiative environments of equal intensity, electron-induced SEU contribution would be totally negligible compared to proton-induced SEU contribution. That means that electron induced SEUs can become significant only in environment largely dominated by these particles.

\section{Gamma-induced SEU}

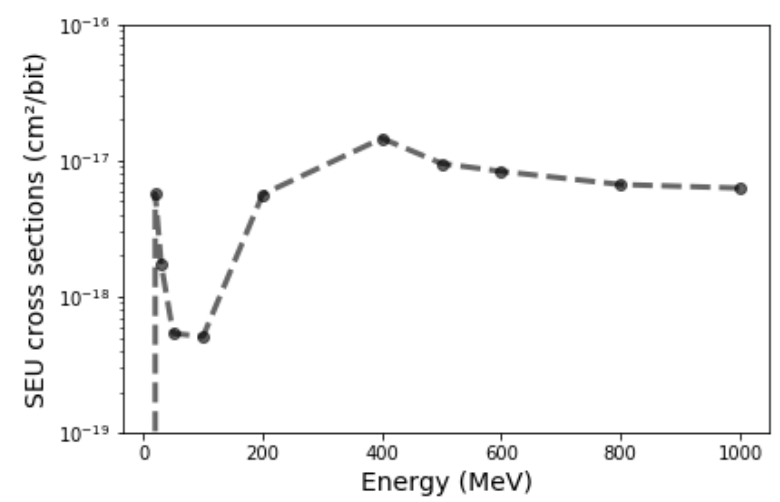

Fig. 3. Predictions of gamma-induced SEU cross sections as a function of incident gamma energies.

Gamma-induced SEU is a second order contribution. Gamma rays are mostly generated by the electrons that cross 
the elements of the satellite. The process then involved is called Bremsstrahlung. Unlike electrons and protons, the probability of generating charges by gamma-induced interaction (photonuclear reaction for instance) must be weighted with the probability of generating gammas by Bremsstrahlung process. Thus, gamma-induced SEU sensitivity study involves two calculation steps:

- Simulation of charge deposition in the device in a gamma environment.

- Simulation of the electron-generated gamma environment due to the Bremsstrahlung process.

The remainder of this section presents the first step, the second step will be addressed in the next section. Gammainduced SEU cross sections have been calculated with our GEANT4 application. Fig. 3 presents our simulated results.

As the critical charge of the IBM device is low, gammainduced SEU cross sections have roughly the same shape of the photonuclear cross sections. Thus, the characteristic regions (Giant Dipole Resonance, ...) of the photonuclear reactions are recognizable [19], [20]. It is already particularly interesting to note that the electron-induced SEU cross sections and gamma-induced SEU cross sections are of the same order of magnitude (see Fig. 2 vs. Fig. 3). That is not what was calculated in [11]. Indeed, the electron-induced SEU cross sections calculated in [11] are not caused by the same physical mechanisms as in our case. Since the investigated device (ESA SEU monitor) has a large critical charge, only electronuclear reactions can produce sufficient charge deposition to trigger upsets. Thus, when the critical charge is large enough, SEU cross sections are mainly driven by nuclear cross sections. Fig. 4 presents the electronuclear and photonuclear cross sections provided by GEANT4 [19], [21].

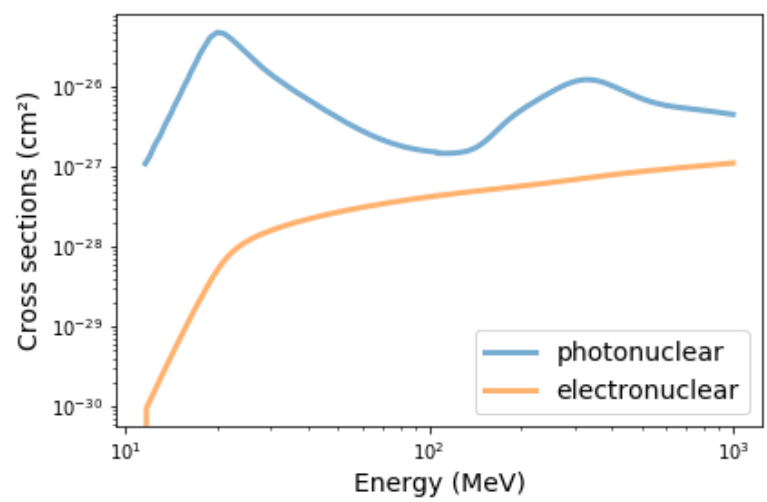

Fig. 4. Photonuclear (blue line) and electronuclear (orange line) cross sections provided by GEANT4.

Of course, in the context of SEU calculations, it is necessary to take into account the probability of depositing a given quantity of charges in the sensitive volume. But Fig. 4 tells us that a gamma is more likely to produce a nuclear reaction than an electron. This explains the results obtained by [11]. In our case (device associated with a low critical charge), the upsets are not driven by the electronuclear reactions but by the Coulomb processes (ionization and elastic scattering). Thus, different conclusions can be expected regarding the importance of primary (protons and electrons) and secondary (gammas) environments in the calculation of upset rates.

\section{ELECTRON-INDUCED SEU DURING EARTH MISSIONS}

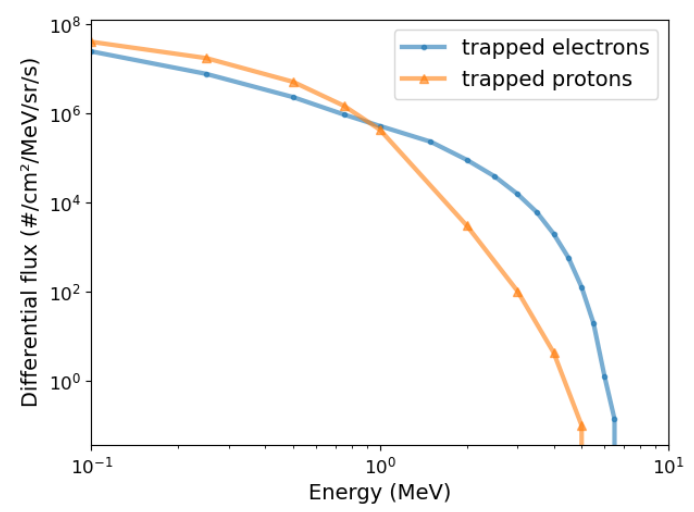

Fig. 5. Differential flux of trapped electrons and protons in GPS orbit calculated with AE8 and AP8 models.

The sensitivities calculated previously can be used to perform some SEU rate calculations by including the ad-hoc environment spectra. In the case of devices associated with a low critical energy, electron-induced ionization process can produce upsets at relatively low electron energies $(<10 \mathrm{MeV})$. The energy range concerned is thus covered by the Earth's electron environments. Therefore, the question of the impact of this type of particle on the in-flight SEU rates may arise. To evaluate the electron sensitivity during Earth mission, the GPS orbit has been chosen. Fig. 5 presents the associated differential fluxes.

Two important pieces of information can be extracted from Fig. 5:

- Available electron energies up to $7 \mathrm{MeV}$

- Maximum proton energy about $5 \mathrm{MeV}$ (which corresponds to a projected range less than $200 \mu \mathrm{m}$ in aluminium [22])

Thus, for the total SEU rate calculation, the trapped protons do not participate because they will be stopped by the satellite's shielding.

As the maximum electron energy is lower than $10 \mathrm{MeV}$, only electron-induced ionization is involved for triggering SEU. Therefore, only devices with a low critical energy can be sensitive to the Earth electron environment.

To properly estimate electron-induced SEU rates, the shielding of the satellite must be taken into account. Two cases are considered here: $2 \mathrm{~mm}$ and $5 \mathrm{~mm}$ aluminium shielding (as presented in Fig. 6).

A dedicated GEANT4 application was developed to evaluate the transported fluxes. However, the dimensions involved are too large to be addressed by discrete models (as provided by the MicroElec module). The standard_opt4 physics list was used instead.

Fig. 6 presents the transported differential fluxes divided between primaries and secondary electrons and photons. Intense photon fluxes can be observed (due to Bremsstrahlung process). Nevertheless, by referring to Fig. 3, the associated energies are too low for triggering upsets (lower than the threshold energy of photonuclear reactions). So, only primary 
and secondary electrons are to be considered. Table II summarizes our electron-induced SEU rate estimations.
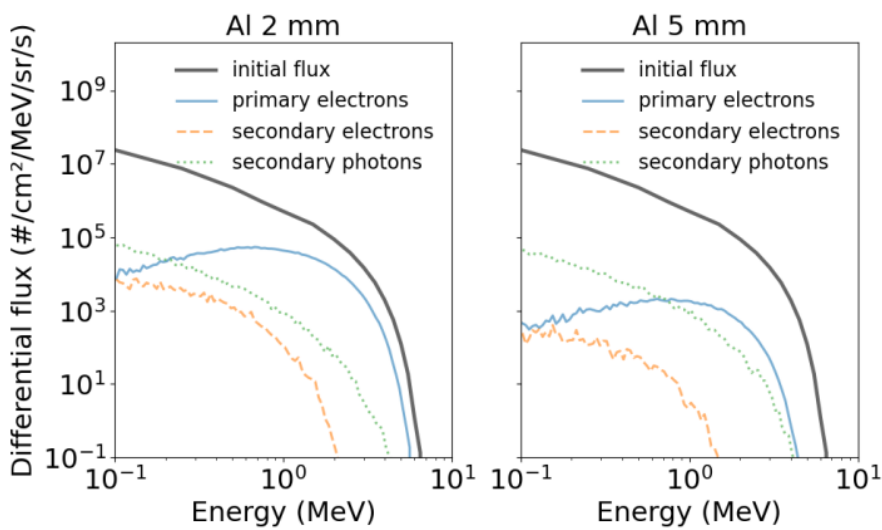

Fig. 6. Transported differential fluxes through $2 \mathrm{~mm}$ of aluminium (left) and 5 mm of aluminium (right) in GPS orbit.

TABLE II

ESTIMATED ELECTRON-INDUCED SEU RATE DURING GPS MISSION FOR DIFFERENT SHIELDING THICKNESSES

\begin{tabular}{|c|c|c|c|}
\hline Shielding (AI) & $\mathbf{0}$ & $2 \mathrm{~mm}$ & $5 \mathrm{~mm}$ \\
\hline SEU rate (\#/bit/year) & $1.98 \cdot 10^{-4}$ & $2.45 \cdot 10^{-5}$ & $9.19 \cdot 10^{-7}$ \\
\hline
\end{tabular}

The case where there is no shielding is given just as an indication. The estimated SEU rates are relatively low but are expressed in per bit and per year. So for a complete device (composed of several million bits), several tens of upsets can be caused by trapped electrons per year of mission.

Finally, even for devices with low critical energies, the Earth electron environment does not seem particularly critical. A much more intense environment will be analyzed in the next section.

\section{ELECTRON-INDUCED SEU DURING JUICE MISSION}

Fig. 7 presents the differential fluences of electrons and protons during JUICE mission extracted from [23].

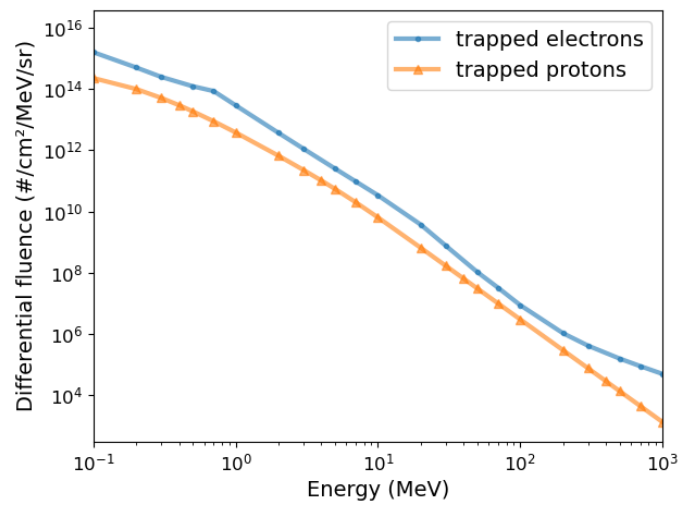

Fig. 7. Differential fluences of trapped electrons and protons during JUICE mission as a function of particle energies.

JUICE mission will be divided in several phases corresponding to interplanetary transfer, transfer to Europa and transfer to Ganymede. As can be noticed by comparing Fig. 5 and Fig. 7, the energies of electrons and protons will be able to reach values that are forbidden around the Earth. That is precisely this energy coverage (up to $\mathrm{GeV}$ ) that has motivated a great deal of interest from the SEU community. Tali et al. [11] have been using differential fluences estimated in [24], but the proton fluxes were underestimated. So our proton induced SEU estimations are expected to be very different from those proposed in [11].

As in the case of GPS missions, it is necessary to take into account the shielding. Here, $20 \mathrm{~mm}$ aluminium shielding is considered. Fig. 8 presents the transported fluences calculated with GEANT4.
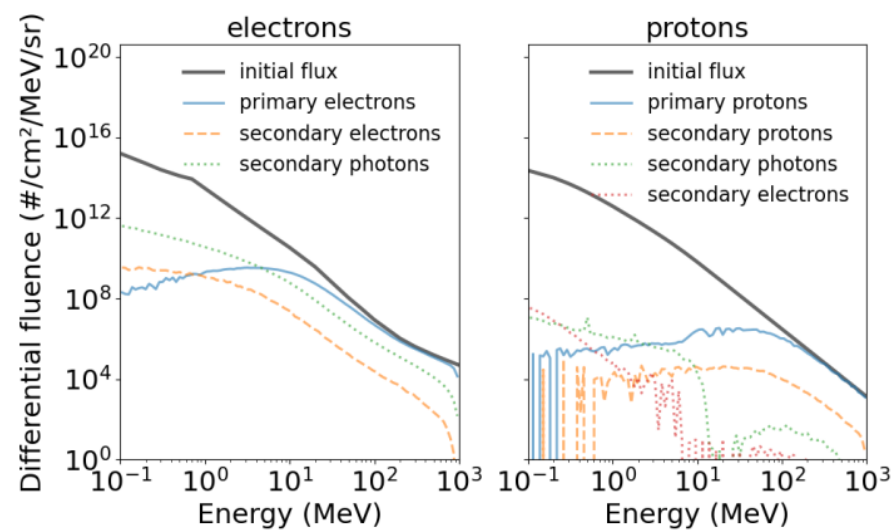

Fig. 8. Transported differential fluences of trapped electrons and protons through $20 \mathrm{~mm}$ of aluminium during JUICE mission as a function of particle energies.

Concerning electron results, large photon fluences can be observed (due to Bremsstrahlung process) and as all the energy of the incident electron can be transferred during this process, photons up to $1 \mathrm{GeV}$ can be generated. Thus, unlike the Earth environment, it is necessary to consider the contribution of secondary photons. Table III summarizes the contribution of each kind of interaction involved.

TABLE III

ESTIMATED ELECTRON-INDUCED SEU RATE DURING JUICE MISSION (TOTAL MISSION). THE MAIN PHYSICAL CONTRIBUTIONS ARE DETAILED

\begin{tabular}{cc}
\hline Contribution & Electron-induced SEU rate $(\# /$ bit/mission) \\
Ionization & $9.70 \cdot 10^{-7}$ \\
Coulomb elastic & $4.02 \cdot 10^{-7}$ \\
Bremsstrahlung & $2.60 \cdot 10^{-8}$ \\
Total & $1.40 \cdot 10^{-6}$ \\
\hline
\end{tabular}

The interesting point is the importance of the Coulomb interactions (inelastic and elastic). Indeed, unlike Tali's conclusions, Bremsstrahlung contribution is negligible compared to the other contributions (identical conclusions are obtained with shielding composed of aluminium and tantalum as proposed in [11]). These differences are due to the sensitivity of the studied device (65-nm SOI SRAM of IBM vs. ESA SEU monitor). As large critical energy is associated 
with the ESA SEU monitor, only nuclear reactions (electronuclear and photonuclear) can generate sufficient deposited energies to trigger upsets.

Our estimation of the total electron-induced SEU rate is about $10^{-6}$ per bit during the whole mission. Again, as a device is composed of several million bits, the number of electron-induced SEU would be of the order of few tens during the JUICE mission. This is an interesting result because our prediction is not so far to the prediction of Tali et al. [11] with the ESA SEU monitor whereas the device studied here is supposed to be more sensitive (lower critical energy). In fact, the decrease of critical energies associated with the increasing integration of the devices is also associated with the decrease of the sensitive volumes. Globally, this means that when particles reach the sensitive volumes, it is easier for them to trigger an upset in integrated memories. Nevertheless, the probability that the incident particles reach the sensitive volumes is lower (since these sensitive volumes are smaller).

Thus, depending what is analyzed, these effects can offset each other. As very high energies are expected during the JUICE mission, nuclear reactions become contributions that need to be accounted for, especially since nuclear reactions are always associated with large deposits of energy. The larger the sensitive volumes, the greater their contributions are likely to be. It is therefore not particularly surprising that an integrated memory presents a sensitivity close to an older memory (i.e. associated with a large technological node) in an environment such as the one encountered by JUICE.

A similar analysis was done concerning the trapped protons and our results are presented in Table IV.

TABLE IV

ESTIMATED PROTON-INDUCED SEU RATE DURING JUICE MISSION (TOTAL MISSION). THE CONTRIBUTIONS BY GENERATED SPECIES THOUGH THE SHIELDING ARE DETAILED

\begin{tabular}{cc}
\hline Contribution & Proton-induced SEU rate $(\# / \mathbf{b i t} / \mathbf{m i s s i o n})$ \\
Protons & $1.59 \cdot 10^{-5}$ \\
Electrons & $2.58 \cdot 10^{-13}$ \\
Photons & $2.29 \cdot 10^{-13}$ \\
Total & $1.59 \cdot 10^{-5}$ \\
\hline
\end{tabular}

Here, the contributions of each species are reported. The generated electrons and photons have contributions that are totally negligible. Our predictions cannot be directly compared to those of Tali et al. [11] since the fluences used are not the same (the proton fluences used here are provided by [23] and are higher than those provided by [24], used by Tali et al. [11]). However, proton-induced SEU rate is roughly one decade greater than electron-induced SEU rate. Thus, for memories associated with low critical energy, the proton environment of the JUICE mission seems to be more critical than the electron environment.

\section{DISCUSSION}

Tali et al. had demonstrated that the contribution of electrons in the total SEU rates during JUICE mission was more important than the contribution of protons [11]. That's not what is found here. Indeed, the fluences evaluated in [24] have been updated in [23]. For a complete comparison between the IBM device (associated with low critical energy) and the ESA SEU monitor (associated with large critical energy), an estimation of the proton-induced SEU rates in the proton environment proposed in [24] was performed. Fig. 9 presents the corresponding transported fluences.

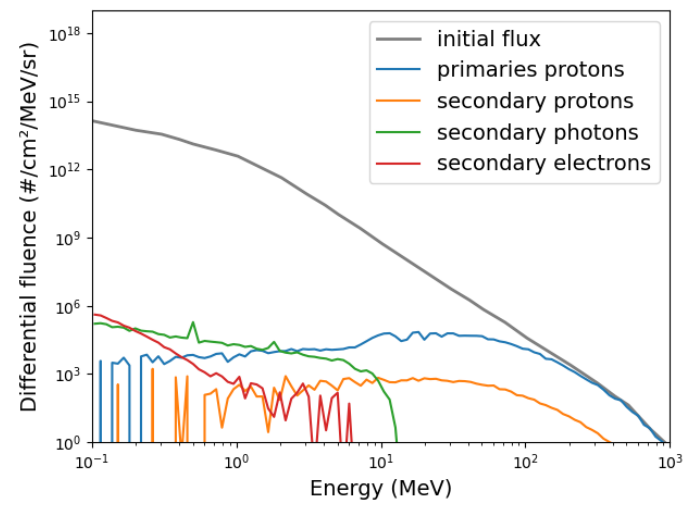

Fig. 9. Transported differential fluences of trapped protons through 20 of aluminium during JUICE mission and using the initial fluence extracted from [24]

Compared to Fig. 8, the fluences in Fig. 9 are significantly lower. It is therefore not particularly surprising that Tali et al. had estimated lower proton-induced SEU rates than those induced by electrons [11]. Table $\mathrm{V}$ presents our estimated SEU rates with the IBM device in this underestimated proton environment.

TABLE V

SAME AS TABLE IV BUT USING PROTON FLUENCE ESTIMATED FROM [24]

\begin{tabular}{cc}
\hline Contribution & Proton-induced SEU rate (\#/bit/mission) \\
Protons & $2.57 \cdot 10^{-7}$ \\
Electrons & $4.09 \cdot 10^{-15}$ \\
Photons & $3.18 \cdot 10^{-16}$ \\
Total & $2.57 \cdot 10^{-7}$ \\
\hline
\end{tabular}

A factor of about 60 can be noted between this result and our previous estimation (see Table IV). In this situation, the electron-induced SEU contribution is more important than the proton-induced SEU contribution (same conclusion as Tali et al.). A ratio of about 6 is obtained between the contributions of electrons and protons while Tali et al. had found a ratio of about 7 . Thus, a very comparable sensitivity can be observed between the IBM device and the ESA SEU monitor (and this, despite the different physical mechanisms involved). 


\section{CONCLUSION}

An analysis of the role of Coulomb processes in electroninduced SEU calculations has been presented. A device with a low critical charge has been selected to investigate the potential consequences of allowable physical mechanisms (ionization and Coulomb elastic scattering). It has been demonstrated that the electron environment of the Earth is now able to trigger upsets due to the low critical energies of the studied device. However, the estimated electron-induced SEU rates seem to be of lesser importance for current space missions. To extend our analysis, the case of the JUICE mission environment has been investigated. In contrast to the conclusions of Tali et al., Bremsstrahlung contributions are found to be negligible compared to Coulomb contributions. This can be explained both by the critical energy of the memory studied here that is lower than the critical energy of the ESA SEU monitor and the dimensions of the sensitive regions that are smaller in the case of the IBM device. In particular, the IBM device seems to have roughly the same sensitivity to the JUICE electron environment compared to the ESA SEU monitor. This underlines that when the environments are very energetic, the dimensions of the sensitive regions of the irradiated device become particularly critical. However, due to the large proton fluence encountered by JUICE, our estimated proton-induced SEU rates are about one decade greater than those induced by electrons.

\section{REFERENCES}

[1] M. P. King et al., "Electron-induced single-event upsets in static random access memory," IEEE Trans. Nucl. Sci., vol. 60, no. 6, pp. 4122-4129, Dec. 2013.

[2] J. M. Trippe et al., "Electron-induced single event upsets in $28 \mathrm{~nm}$ and $45 \mathrm{~nm}$ bulk SRAMs," IEEE Trans. Nucl. Sci., vol. 62, no. 6, pp. 27092716, Dec. 2015.

[3] A. Samaras et al., "Experimental characterization and simulation of electron-induced SEU in 45-nm CMOS technology," IEEE Trans. Nucl. Sci., vol. 61, no. 6, pp. 3055-3060, Dec. 2014.

[4] M. J. Gadlage, A. H. Roach, A. R. Duncan, M. W. Savage, and M. J. Kay, "Electron-induced single-event upsets in 45-nm and 28-nm bulk CMOS SRAM-based FPGAs operating at nominal voltage," IEEE Trans. Nucl. Sci., vol. 62, no. 6, pp. 2717-2724, Dec. 2015.

[5] M. J. Gadlage, A. H. Roach, A. R. Duncan, A. M. Williams, D. P. Bossev, and M. J. Kay, "Soft errors induced by high-energy electrons," IEEE Trans. Device Mater. Reliab., vol. 17, no. 1, pp. 157-162, Mar. 2017.

[6] M. J. Gadlage, A. H. Roach, A. R. Duncan, A. M. Williams, D. P. Bossev, and M. J. Kay, "Multiple-cell upsets induced by single highenergy electrons," IEEE Trans. Nucl. Sci., vol. 65, no. 1, pp. 211-216, Jan. 2018.

[7] C. Inguimbert, R. Ecoffet, and D. Falguere, "Electron induced SEUs: microdosimetry in nanometric volumes," IEEE Trans. Nucl. Sci., vol. 62, no. 6, pp. 2846-2852, Dec. 2015.

[8] P. Caron et al., "Physical mechanisms inducing electron single-event upset," IEEE Trans. Nucl. Sci., vol. 65, no. 8, pp. 1759-1767, Aug. 2018.

[9] A. Akkerman, J. Barak, and N. M. Yitzhak, "Role of elastic scattering of protons, muons, and electrons in inducing single event upsets," IEEE Trans. Nucl. Sci., vol. 64, no. 10, pp. 2648-2660, Aug. 2017.

[10] P. Caron, C. Inguimbert, L. Artola, F. Bezerra, and R. Ecoffet, "New SEU modeling method for calibrating target system to multiple radiation particles," IEEE Trans. Nucl. Sci., vol. 67, no. 1, pp. 44-49, Jan. 2020.

[11] M. Tali et al., "High-energy electron-induced SEUs and Jovian environment impact," IEEE Trans. Nucl. Sci., vol. 64, no. 8, pp. 20162022, Aug. 2017.

[12] D. F. Heidel et al., "Low energy proton single-event-upset test results on 65 nm SOI SRAM," IEEE Trans. Nucl. Sci., vol. 55, no. 6, pp. 33943400, Dec. 2008.
[13] S. Agostinelli et al., "Geant4-a simulation toolkit," Nucl. Instrum. Methods Phys. Res. Sect. Accel. Spectrometers Detect. Assoc. Equip., vol. 506, no. 3, pp. 250-303, Jul. 2003.

[14] J. Allison et al., "Geant4 developments and applications," IEEE Trans. Nucl. Sci., vol. 53, no. 1, pp. 270-278, Feb. 2006.

[15] P. Caron, C. Inguimbert, L. Artola, R. Ecoffet, and F. Bezerra, "Physical mechanisms of proton-induced single-event upset in integrated memory devices," IEEE Trans. Nucl. Sci., vol. 66, no. 7, pp. 1404-1409, Jul. 2019.

[16] N. A. Dodds et al., "New insights gained on mechanisms of low-energy proton-induced SEUs by minimizing energy straggle," IEEE Trans. Nucl. Sci., vol. 62, no. 6, pp. 2822-2829, Dec. 2015.

[17] A. Valentin, M. Raine, J.-E. Sauvestre, M. Gaillardin, and P. Paillet, "Geant 4 physics processes for microdosimetry simulation: Very low energy electromagnetic models for electrons in silicon," Nucl. Instrum. Methods Phys. Res. Sect. B Beam Interact. Mater. At., vol. 288, pp. 6673, Oct. 2012.

[18] A. Valentin, M. Raine, M. Gaillardin, and P. Paillet, "Geant4 physics processes for microdosimetry simulation: very low energy electromagnetic models for protons and heavy ions in silicon," Nucl. Instrum. Methods Phys. Res. Sect. B Beam Interact. Mater. At., vol. 287, pp. 124-129, Sep. 2012.

[19] M. V. Kossov, "Approximation of photonuclear interaction crosssections," Eur. Phys. J. - Hadrons Nucl., vol. 14, no. 3, pp. 377-392, Jul. 2002.

[20] J. Ahrens et al., "Total nuclear photon absorption cross sections for some light elements," Nucl. Phys. A, vol. 251, no. 3, pp. 479-492, Oct. 1975.

[21] J. P. Wellisch, M. Kossov, and P. Degtyarenko, "Electro and gamma nuclear physics in Geant4," ArXivnucl-Th0306012, Jun. 2003, Accessed: Sep. 23, 2017. [Online]. Available: http://arxiv.org/abs/nuclth/0306012.

[22] J. F. Ziegler, M. D. Ziegler, and J. P. Biersack, "SRIM - The stopping and range of ions in matter (2010)," Nucl. Instrum. Methods Phys. Res. Sect. B Beam Interact. Mater. At., vol. 268, no. 11-12, pp. 1818-1823, Jun. 2010.

[23] JUICE Team, "JUICE environment specification," ESTEC/ESA Noordwijk, The Netherlands, Tech. Rep. JS-14-09, 2017.

[24] JUICE Team, "JUICE environment specification," ESTEC/ESA Noordwijk, The Netherlands, JS-14-09, 2015. 